\title{
3D Simulation of Plume Flows from a Cluster of Plasma Thrusters
}

\author{
Chunpei Cai ${ }^{*}$ and Iain D. Boyd ${ }^{\dagger}$ \\ Department of Aerospace Engineering \\ University of Michigan, Ann Arbor, MI 48109
}

In this study, a 3D simulation of xenon plasma plume flow fields from a cluster of four Hall thrusters is performed with a hybrid particle-fluid method. In this simulation, a detailed fluid model is used to compute the electron properties, the direct simulation Monte Carlo method models the collisions of heavy particles and the Particle In Cell method models the transport of ions in electric fields. The accuracy of the simulation is assessed through comparisons with available measured data. The simulation successfully captures detailed plume structures and plume interactions.

\section{Nomenclature}

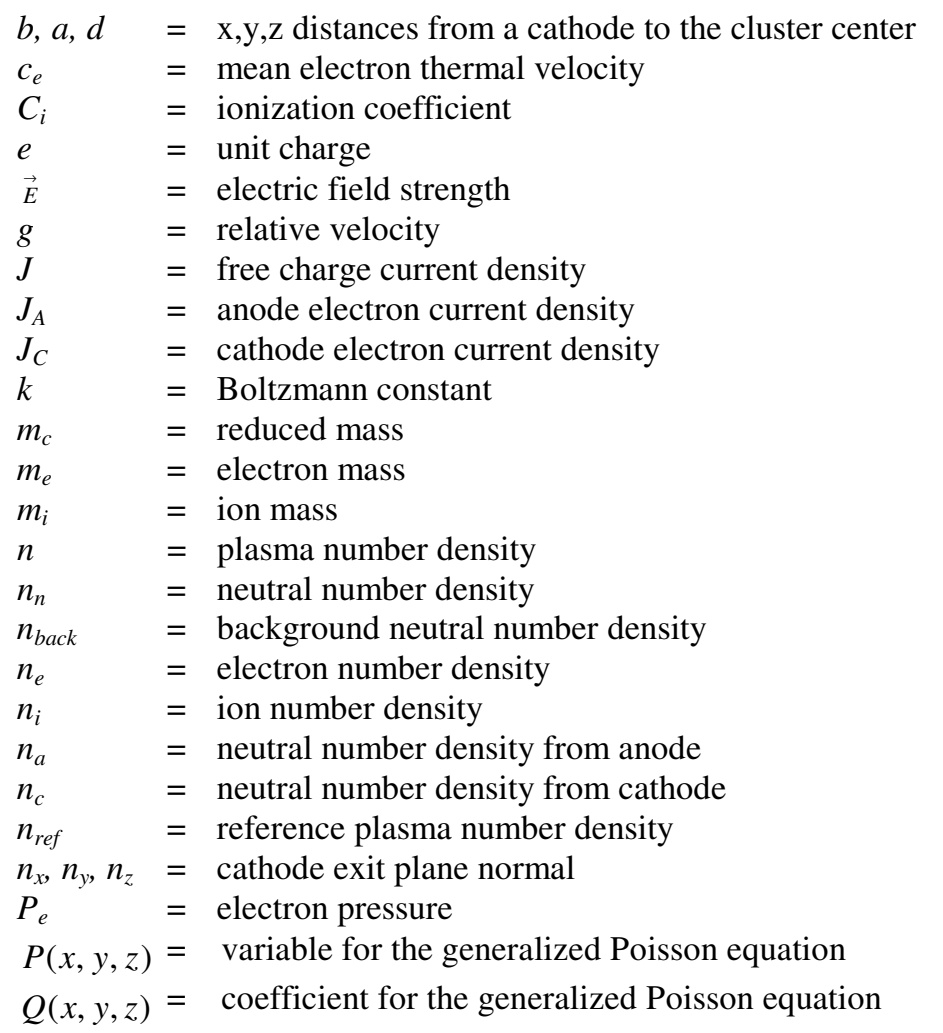

\footnotetext{
* Graduate Student, AIAA student member (ccai@engin.umich.edu).

${ }^{\dagger}$ Professor, AIAA associate fellow (iainboyd@umich.edu).

Copyright $(92005$ by the American Institute of Aeronautics and Astronautics, Inc. All Right Reserved.
} 


$\begin{array}{lll}\vec{r} & =\text { distance vector between two points } \\ R_{c} & =\text { cathode orifice radius } \\ S(x, y, z) & =\text { source term for the generalized Poisson equation } \\ T e & =\text { electron temperature } \\ T e_{r e f} & =\text { reference electron temperature } \\ T_{H} & =\text { heavy particle temperature } \\ V_{e} & =\text { electron velocity vector } \\ V & =\text { cell volume } \\ \sigma & =\text { plasma conductivity } \\ \sigma_{i} & =\text { reference cross section for xenon } \\ \theta_{+} & =\text {divergence angle for the upper channel edge } \\ \theta & =\text { divergence angle for the lower channel edge } \\ \boldsymbol{\kappa}_{e} & =\text { electron thermal conductivity } \\ \mathcal{E}_{i} & =\text { ionization energy } \\ \phi & =\text { plasma potential } \\ \phi_{r e f} & =\text { reference plasma potential } \\ \omega & =\text { viscosity temperature exponent } \\ \Omega & =\text { solid angle in velocity phase space } \\ v_{e} & =\text { electron collision frequency, } v_{e}=v_{e i}+v_{e n} \\ v_{e i} & =\text { ion-electron collision frequency } \\ v_{e n} & =\text { neutral electron collision frequency } \\ \psi & =\text { electron stream function }\end{array}$

\section{Introduction}

$\mathrm{H}$ ALL thrusters represent a very efficient form of electric propulsion devices widely used on spacecraft for onorbit applications such as station keeping. In general, Hall thrusters are replacing chemical thrusters in specific applications because of several merits. Hall thrusters can create higher specific impulse, obtain electricity input directly in space through solar cells, and do not require carrying oxidant. High power electric propulsion systems are being investigated due to improvements in solar cell technology and due to renewed interest in nuclear power.

The development of high-power Hall thrusters falls into two categories: one case involves investigating single, monolithic thrusters, while the second case involves clustering several small thrusters. Generally, clustering is favorable because of several merits including a cheaper manufacturing cost, less demanding requirement from test facilities, more robustness and ability to tolerate failure of single thrusters.

There are several major interests in numerical simulation of plasma flows from a cluster of Hall thrusters. One interest is to investigate the plume interactions, especially in the complex and important near field locations. The performance of a thruster in a cluster may be different from a standalone situation. Another interest is to estimate plume impingement, which involves high-energy ions and charge exchange ions (CEX), onto sensitive spacecraft surfaces such as solar arrays. When a fast ion collides with a slow neutral, one or two electrons may transfer from the neutral to the ion, resulting in a slow ion and a fast neutral. Under the electric field, this ion may drift behind the thruster. Severe impingement of ions onto spacecraft surfaces may result eventually in failure of devices or even a final failure of the whole mission. If severe impingement is predicted, then a change of design philosophy must be considered to reduce the impingement.

To accurately simulate the plasma plumes from a cluster of Hall thrusters, an accurate modeling of the complex physical plume mechanism is required. A plasma plume is a complex rarefied flow with several species: atoms, positively charged ions and negatively charged electrons. Traditionally, the computational simulation of plasma plume flows into vacuum is performed with a hybrid particle-fluid approach. The direct simulation Monte Carlo (DSMC) method ${ }^{1}$ models the collisions of the heavy particles (ions and atoms) while the Particle In Cell (PIC) method $^{2}$ models the transport of the ions in electric fields. The electrons are modeled using a fluid description because they can adjust their velocities more quickly with their significantly lighter mass.

For the fluid electron model, the Boltzmann relation is usually adopted to compute the plasma potential. The Boltzmann relation has many assumptions such as a constant electron temperature distribution. A recently proposed 
detailed fluid electron model by Boyd and $\mathrm{Yim}^{3}$ was based on the conservation laws for electrons and is capable of providing accurate and detailed distributions for electron temperature, plasma potential and electron velocity. This model was successfully applied in a simulation of an axi-symmetric plasma plume from a 200W class Hall thruster.

In this study, the 3D plume flow fields from a cluster of four 200W class Hall thrusters are simulated with the DSMC-PIC methods and the detailed fluid electron model. It is significant to mention that this is the first time the detailed model is applied in a 3D situation.

Section II briefly introduces background information from experiments; section III reviews the general DSMCPIC hybrid methods with several numerical implementation issues, and finally section IV presents a 3D simulation and discussion of results.

\section{Background}

The devices considered in the present study are a cluster of four BHT-200 Hall thrusters manufactured by Busek, Co. Each thruster is operated at $200 \mathrm{~W}$ with a nominal thruster level of $13 \mathrm{mN}$. The cluster of BHT-200 thrusters has been investigated experimentally., ${ }^{4,5}$

The data in Ref. 5 were taken in a 9m by $6 \mathrm{~m}$ tank of the Plasmadynamics \& Electric Propulsion Laboratory (PEPL) at the University of Michigan and Fig. 1 is a photograph of the thrusters in operation. These four thrusters are configured in a 2 by 2 grid with a center-to-center distance of $0.115 \mathrm{~m}$. In the following simulation, the same thruster numbering as Ref. 5 is adopted: the upper left, lower left, lower right and upper right thrusters are named as thruster 1, 2, 3, 4. As shown in the same photograph, there are four cathode-neutralizers located either above or at the bottom of each thruster, and there is a $7 \mathrm{~mm}$ conic cap on the front face of each thruster to protect the thruster against ion sputtering. Due to the symmetry, only one thruster and one cathode are needed in the 3D simulation.

The total pumping speed in this facility for these experiments was $140,000 \mathrm{l} / \mathrm{s}$ on xenon resulting in a backpressure of $1.1 \times 10^{-6}$ Torr. Intrusive Faraday probes were used to measure angular profiles of ion current density. A retarding potential analyzer was also used to measure the ion energy distribution function in the plume far field. In addition, a floating emissive probe and a triple Langmuir probe were used to measure the plasma potential, the electron number density and the electron temperature.

Xenon is usually adopted for plasma propellant. A

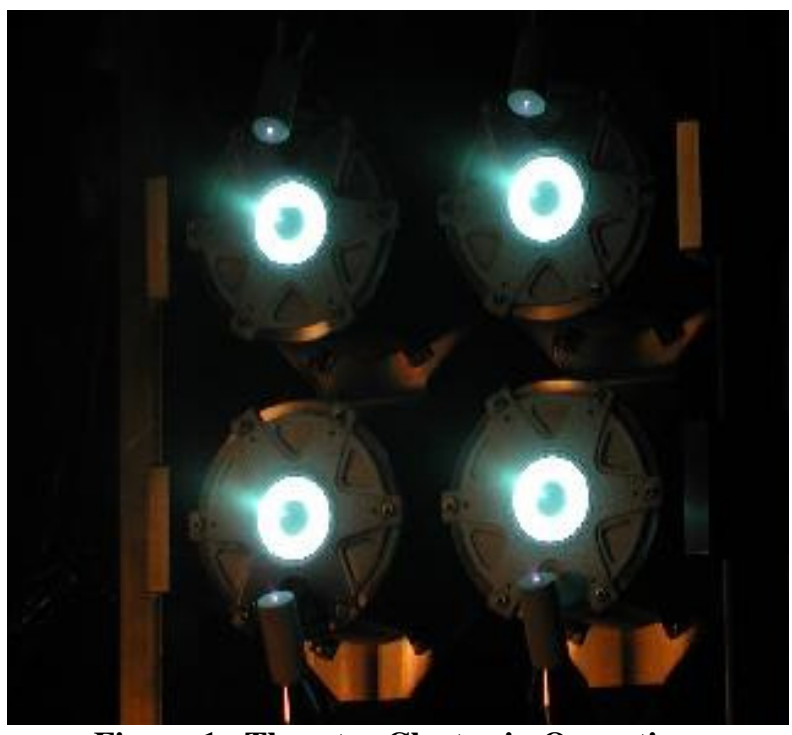

Figure 1. Thruster Cluster in Operation. (Courtesy of PEPL) plume from a plasma thruster consists of light electrons with speeds of $1 \times 10^{6} \mathrm{~m} / \mathrm{s}$, and heavier ions or neutrals, such as fast single or double charged ions, $\mathrm{Xe}^{+}, \mathrm{Xe}^{++}$, slow neutral xenon, and fast neutral xenon and slow ions due to CEX.

\section{Simulation Methods and Numerical Implementation Issues}

\section{General Steps for the DSMC-PIC Methods}

For particle simulations of plasma plume flow, heavy neutrals and ion particles are simulated with the DSMC and the PIC methods, while the electrons are modeled as a fluid because electrons can adjust themselves more quickly. The DSMC-PIC simulation can be summarized with the following steps:

Step 1. (PIC): Allocate the charge of each ion inside a cell onto the cell nodes.

Step 2. (Fluid): Calculate plasma potential $\phi$ using a fluid electron model.

Step 3. (PIC): Calculate ionization in all cells. A fraction of neutrals will be changed to ions.

$$
d n_{i}=C_{i} n_{n} n_{i}
$$

Step 4. (PIC): Calculate the electric field on each node with the relation:

$$
\vec{E}=-\nabla \phi
$$


Step 5. (DSMC, PIC): Sample quantities inside each cell.

Step 6. (DSMC): Perform momentum change and CEX collisions inside each cell.

Step 7. (DSMC, PIC): Introduce new particles (ions and neutrals) into the simulation domain from inlet boundaries.

Step 8. (PIC): Calculate the ion acceleration based on its location inside its cell.

Step 9. (DSMC, PIC): Move all particles with the time step.

In this study, magnetic field effects are neglected.

\section{The Detailed Fluid Electron Model}

In Step 2, the simplest fluid electron model is the Boltzmann relation, which is obtained from the electron momentum equation:

$$
\phi=\phi_{r e f}+\frac{k T_{r e f}}{e} \ln \left(\frac{n_{e}}{n_{r e f}}\right)
$$

This equation is derived using several strong assumptions. These assumptions include that the fluid electron flow is isothermal, collisionless, the electron pressure obeys the ideal gas law and the magnetic field is neglected. However, in plasma plumes, especially in the near field, there are significant gradients and the approximation may be inappropriate.

To improve the understanding of the plume flow characteristics, recently a detailed electron model was proposed $^{3}$ and illustrated with an axi-symmetric plume simulation. The major results in Ref. (3) are summarized here for reference.

In the detailed model, an equation for the electron stream function $\Psi$ can be deduced from the steady mass conservation law for electrons and expressed as:

$$
\nabla^{2} \psi=C_{i} n_{e} n_{n}
$$

where $\nabla \psi=n_{e} \vec{V}_{e}$.

The xenon ionization rate coefficient is expressed as a function of electron temperature using a simple relation proposed by Ahedo: ${ }^{6}$

From the generalized Ohm's law:

$$
C_{i}=\sigma_{i} c_{e}\left(1+\frac{T_{e} \varepsilon_{i}}{\left(T_{e}+\varepsilon_{i}\right)^{2}}\right) \exp \left(-\frac{\varepsilon_{i}}{T_{e}}\right)
$$

$$
\vec{j}=\sigma\left[-\nabla \phi+\frac{1}{e n_{e}} \nabla\left(n_{e} k T_{e}\right)\right]
$$

an equation for electron potential $\phi$ is obtained:

$$
\nabla \bullet(\sigma \nabla \phi)=\frac{k \sigma}{e}\left(\nabla^{2} T_{e}+T_{e} \nabla^{2}\left(\ln \left(n_{e}\right)\right)+\sigma \nabla\left(\ln n_{e}\right) \bullet \nabla T_{e}+T_{e} \nabla \sigma \bullet \nabla\left(\ln \left(n_{e}\right)+\nabla \sigma \bullet \nabla T_{e}\right)\right.
$$

The electron temperature equation is obtained from the steady state electron energy equation:

$$
\nabla^{2} T_{e}=-\nabla \ln \left(\kappa_{e}\right) \bullet \nabla T_{e}+\frac{1}{\kappa_{e}}\left(-j \bullet E+\frac{3}{2} n_{e}(\vec{V} \bullet \nabla) k T_{e}+p_{e} \nabla \bullet \vec{V}_{e}+3 \frac{m_{e}}{m_{i}} v_{e} n_{e} k\left(T_{e}-T_{H}\right)+n_{e} n_{a} C_{i} \varepsilon_{i}\right)
$$

The electron number density $n_{e}$ is set equal to the ion number density $n_{i}$ based on the plasma quasi-neutral condition. The electron conductivity $\sigma$, the electron thermal conductivity $\kappa_{e}$, the ion-electron collision frequency $v_{e i}$, and the neutral electron collision frequency $v_{e n}$ can be found in Ref. 7 and its references:

$$
\begin{gathered}
\sigma=\frac{e^{2} n_{e}}{m_{e} v_{e}} \\
\kappa_{e}=\frac{2.4}{1+\frac{v_{e i}}{\sqrt{2 v_{e}}}} \frac{k^{2} n_{e} T_{e}}{m_{e} V_{e}}
\end{gathered}
$$

By treating the right hand side terms as known sources and solving Eqs. $(4,7,8)$, three fundamental electron properties are obtained, i.e., electron velocity, plasma potential, and electron temperature. With these detailed properties, the plasma plume simulation yields much improved results in comparison to the Boltzmann relation. 
As a continuing effort, this paper presents the recent progress of applying the detailed electron model in a 3D situation on an unstructured mesh. The plume flows from a cluster of four thrusters into vacuum are simulated with the DSMC-PIC methods and this detailed electron model.

\section{General Finite Element Solver for Poisson Equations}

Each of equations $(4,7,8)$ can be expressed as a general Poisson equation ${ }^{8}$ :

$$
\nabla \bullet(Q(x, y, z) \nabla P(x, y, z))=S(x, y, z)
$$

where $Q(x, y, z)$ is a distribution of coefficients, $P(x, y, z)$ is a distribution of the primary variable to be solved and $S(x, y, z)$ is a known source term.

For a two-dimensional or axi-symmetric simulation on a structured mesh, an ADI iterative solver is usually adopted for simplicity. However, there are several drawbacks for the ADI method:

1). It is not Not applicable on unstructured meshes.

2). For a structured mesh, depending on the geometry of the simulation, the ADI method needs to be applied on each sub-domain separately with artificial inner boundaries and the artificial inner boundaries may result in inaccuracy in the simulation results. This precludes the application of ADI to complex geometries, even with structured meshes.

3). The treatment of boundaries is not natural. This may result in inaccuracies for the source terms on the first layer of nodes at the boundary. At the thruster exit, the gradients are significant and a mistreatment of boundary source conditions will spread the effects into the whole flow domain because of the elliptical property of these equations. The first three layers of nodes close to the boundaries will be heavily affected.

To simulate flows with very complex geometry, an unstructured mesh must be adopted and this actually precludes the ADI method.

To address the above problems, a general purpose finite element solver applicable to two- and three-dimensional structured and unstructured meshes is developed to solve the above three equations. Compared with the ADI method, the finite element method is applicable on unstructured meshes and can integrate the boundary conditions more accurately. The discrete stiff matrix for a triangular cell can be found in Ref. 8, and a quadrilateral cell can be considered as two triangles. For a 3D tetrahedral cell with volume $\mathrm{V}$ and node coordinates of $\left(x_{i}, y_{i}, z_{i}\right), i=1,2$, 3,4 , after discretization the stiff matrix is:

where

$$
\left[B_{e}^{T} B_{e}\left\{Q_{a v e} P_{n e}\right\}-\frac{1}{20}\left[\begin{array}{cccc}
2 & 1 & 1 & 1 \\
1 & 2 & 1 & 1 \\
1 & 1 & 2 & 1 \\
1 & 1 & 1 & 2
\end{array}\right]\left\{S_{n e}\right\}\right]=0
$$

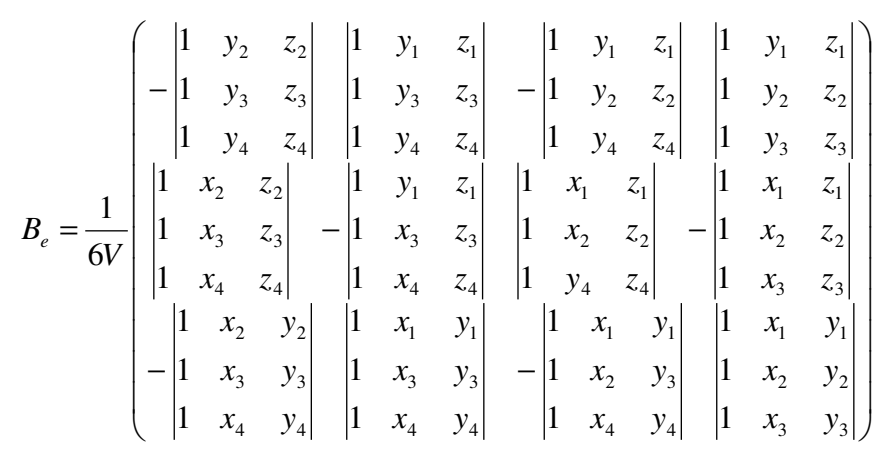

The final stiff matrix is sparse, symmetric and positive definite. These novel properties are not naturally guaranteed by a finite difference method or a finite volume method. To fully take advantage of these properties, an efficient storage scheme is adopted which only requires a cost of $O(n)$ where $n$ is the total node number. The iterative conjugate gradients method ${ }^{9}$ is adopted to solve the final linear equations. This method fully takes advantage of the symmetric and positive definite properties of the stiff matrix. 


\section{Derivative Calculation on an Unstructured Mesh}

Several situations will require the calculation of derivatives on a node. Two situations are Step 2 to calculate the source terms for Eqs. $(4,7,8)$ and Step 3 to calculate the electric field from a potential field. Besides accuracy, one requirement for the optimal calculation scheme is to be applicable on both serial and parallel machines.

There are several options to calculate derivatives on unstructured meshes. One of them is the least square method which can be found in Ref. 10. The basic idea of the least square method can be illustrated with an example to calculate the electric field from a potential field: assume the unknown gradients on one node are $E(x, y, z)=\left(E_{x}, E_{y}, E_{z}\right)$. If there are $\mathrm{N}$ nodes connected to this node with differences of value $(d \phi)_{i}$ and distance vectors $(d X)_{i}$, then they will form $\mathrm{N}$ by 3 relations which are over determined:

$$
M E=d \Phi
$$

where $M$ is an $\mathrm{N} \times 3$ matrix, $E$ is a $3 \times 1$ vector, and $d \Phi$ is an $\mathrm{N} \times 1$ vector. By multiplying by a transposed matrix $M^{T}$ on both sides, this over determined matrix is transformed to a $3 \times 3$ matrix and the equations are solvable.

One tetrahedral cell is enough to decide the derivatives on a specific node. This scheme will include the effects from all nodes connected to a specific node hence it will yield an accurate result. The least square method is also applicable on parallel machines. The only extra cost of this scheme is, at the beginning of a simulation, the node connection relations must be gathered and saved for the whole simulation process.

\section{Weighting Scheme}

The weighting scheme is critical for a successful DSMC-PIC simulation with the detailed fluid electron model. In Step 1, the ion number density at a specific node must be accurately estimated by weighting the charge of ions in all cells connected with this node. In Step 8, the acceleration for a particle is interpolated from the values on the same nodes. Generally these two weighting schemes are preferably the same, and the closer a particle to a node, the more influence this particle will have on or from the node.

Usually for this charge allocation, a weighting
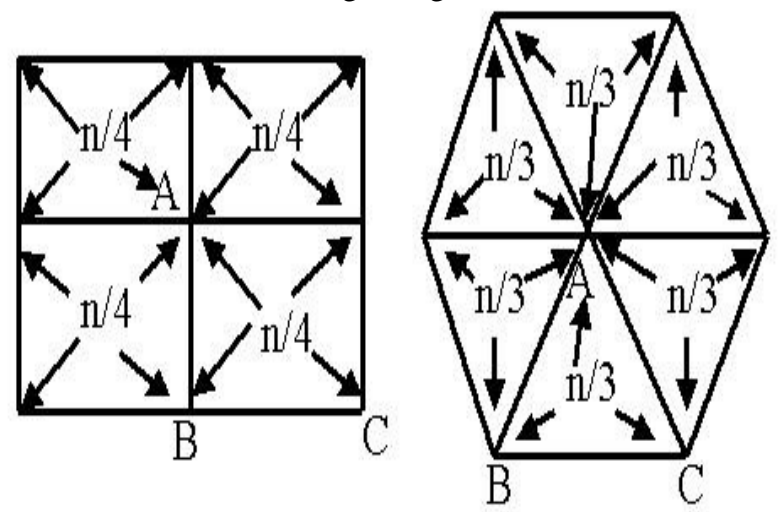

Figure 2. Illustration of charge allocation effects. scheme based on areas or volumes is adopted. For example, Ruyten ${ }^{11}$ presented a well-used scheme for structured axi-symmetric meshes. In the literature, there are reports that weighting schemes based on areas or volumes are applied for unstructured axi-symmetric and three-dimensional situations as well. However, it can be shown that for an unstructured mesh it is improper to allocate charges with a scheme based on areas or volumes.

Suppose there are a large number of particles in each cell and the average number density is $n$. Weighting by cell area or volume will equally distribute the ion density onto the nodes forming this cell. Consider Fig. 2, for a twodimensional mesh, on the left, the center node A is shared by four cells, and the total charge number density at the center node $\mathrm{A}$ is $n / 4 \times 4=n$. While for the unstructured mesh on the right, the total charge number density will be $n / 3 \times 6=2 n$. While for $3 \mathrm{D}$ unstructured tetrahedral cells, usually there are about 16 cells connected to an inner node, hence the number density at that node will be calculated as $n / 4 \times 16=4 n$. It is obvious that for an unstructured mesh if the weighting scheme by areas or volumes is applied, for 2D with triangles the charge density at one node will be two times higher than its real value, while for an unstructured 3D situation, the estimated charge density will be four or five times higher than the real charge density, depending on how many neighboring nodes are connected to this specific node.

Another problem is associated with the weighting scheme by areas or volumes. In Fig. 2, the number density at a boundary node may be incorrect. For example, if node B in the left picture is on a boundary, then the number density on node $B$ will be $n / 4 \times 2=n / 2$. Compensations will be necessary to correct the number density for the boundary nodes.

If the Boltzmann relation is used to compute the plasma potential, this overestimated charge density may not result in any significant problems. The mistake in plasma potential is offset by the log function in Eq. (3), and this error is further cancelled in the calculation of the electric field by Eq. (2). As a result, even though the number density and the plasma potential are not calculated correctly by the weighting scheme of areas or volumes, the electric field is almost correct. This is the possible reason why there are few reports of this problem in the literature. 
For the detailed fluid electron model, which includes detailed physical terms and the potential is calculated by Eq.(7), this mistake will create a false potential field.

For the ionization source term in Eq. (1), both the charge density and the neutral density on a node will be over estimated, and a minimum of $4 \times 4-1=15$ times higher source will be calculated for the ionization in Step 2. In the near field, the neutral number density is usually much higher than the ion number density; hence an overestimated ionization rate will create a positive feedback for the ion number density. Finally, in the near field, a false high accumulation of ion number density will be generated. For the detailed fluid electron model, where the potential calculation is more sensitive than the Boltzmann relation, the weighting scheme by areas or volume will trigger the chain reaction in ionization and finally invalidate the simulation.

In Ref. 10, one scheme is reported which requires a high quality mesh. The total charge of a particle is assigned to the closest node from this particle. Though this scheme is physically accurate, there are two problems. The first problem is there are situations when the nearest node to the particle is not one of the cell nodes forming the cell where the particle is located, such as regions near boundaries with boundary constrains; the second problem is this scheme will require many cross nodes transportations on a parallel machine and it will be quite inefficient.

The charge and neutral allocation scheme adopted in this study is a simple one. First the cell average values are calculated and then these values are averaged onto the nodes. Suppose there are $N$ cells connected to a node, and the $j$ th cell has an averaged charge density $n_{j}$, then the charge density $n$ on the node can be expressed as:

$$
n=\left(\sum_{j=1}^{N} n_{j}\right) / N
$$

This scheme is efficient on a parallel machine without significant loss of accuracy. The interpolation of electric field with the weighting scheme by areas or volumes is still correct and no change is necessary.

\section{Collision Dynamics}

The DSMC method uses particles to simulate collision effects in rarefied gas flow by collecting groups of particles into cells which have size of the order of a mean free path. In Step 6, pairs of particles inside a cell are selected at random and a collision probability is evaluated that is proportional to the product of the relative velocity and the collision cross section for each pair. The probability is computed with a random number to determine if that collision occurs. If so, some form of collision dynamics is performed to alter the properties of the colliding particles. The no time counter method ${ }^{1}$ is adopted to determine if a collision occurs in this study.

There are two types of collisions that are important in these Hall thruster plumes: elastic (momentum exchange) and charge exchange (CEX). The elastic collisions involve only exchange of momentum between the participating particles. For atom-atom collisions, the Variable Hard Sphere ${ }^{1}$ model is employed and the collision cross section of xenon is:

$$
\sigma_{e l}(\mathrm{Xe}, \mathrm{Xe})=\frac{2.12 \times 10^{-18}}{g^{2 \omega}} m^{2}
$$

where $g$ is the relative velocity and $\omega=0.12$ is related to the viscosity temperature exponent for xenon. For atomion elastic interactions, the following cross section of Dalgarno ${ }^{12}$ et al is employed:

$$
\sigma_{e l}\left(\mathrm{Xe}_{\mathrm{Xe}} \mathrm{Xe}^{+}\right)=\frac{6.42 \times 10^{-18}}{g} m^{2}
$$

In all elastic interactions, the collision dynamics is modeled using isotropic scattering together with conservation of linear momentum and energy to determine the post-collision velocities of the colliding particles.

Charge exchange concerns the transfer of one or more electrons between an atom and an ion. When a CEX collision happens between fast ion collides with a slow neutral, their velocity exchange as well. For singly and doubly charged ions, the following cross section measured by Pullins et al. ${ }^{13}$ and Miller et al. ${ }^{14}$ are used:

$$
\begin{gathered}
\sigma_{c e x}\left(\mathrm{Xe}, \mathrm{Xe}^{+}\right)=2.0 \times 10^{-20}\left(87.3-13.6 \log \left(\frac{m_{c} g^{2}}{2}\right)\right) \\
\sigma_{c e x}\left(\mathrm{Xe}, \mathrm{Xe}^{2+}\right)=2.0 \times 10^{-20}\left(45.7-8.9 \log \left(\frac{m_{c} g^{2}}{2}\right)\right)
\end{gathered}
$$

where $m_{c}$ is the reduced mass. It is assumed that there is no transfer of momentum accompanying the transfer of the electron(s). This assumption is based on the premise that charge exchange interactions are primarily at long range. 


\section{Boundary Conditions}

When an ion particle hits a wall, it loses its charge and reflects diffusely as a neutral particle with a thermal velocity for a wall temperature of $300 \mathrm{~K}$. For the thrusters in this study, the front wall is dielectric, and the sheath voltage is significant. The sheath voltage for the front wall can be estimated by a transformation of Eq. (6):

$$
\phi_{w}=\phi_{0}+\left(\frac{\vec{J}}{\sigma}-\frac{k \nabla\left(n_{e} T_{e}\right)}{e n_{e}}\right) \bullet d l
$$

where $\phi_{0}$ is the potential at the node next to the wall.

For other wall locations, a potential of $0 \mathrm{~V}$ is appropriate. The gradient of electron temperature is set to zero at each wall.

A neutral background gas at a pressure $1 \times 10^{-3} \mathrm{~Pa}$ and a temperature of $300 \mathrm{k}$ is maintained in the simulation.

Several macroscopic properties of the plasma are required for the computations. Specifically, the plasma potential, the electron stream function and the electron temperature are required for all boundaries. At an inlet, for all heavy species, the number density, velocity and temperatures are required. The setup employs a mixture of analysis and estimation based on experimental data of the mass flow rate from anodes and cathodes, thrust, and total ion current. The neutrals are assumed to exit the thruster and cathodes at the sonic speed corresponding to assumed values for their temperature. Finally a divergence angle of $\theta=30$ degree for the lower edge and $\theta_{+}=20$ degree for the upper edges of the exit channel are assumed. Table 1 summarizes the boundary conditions for the stream

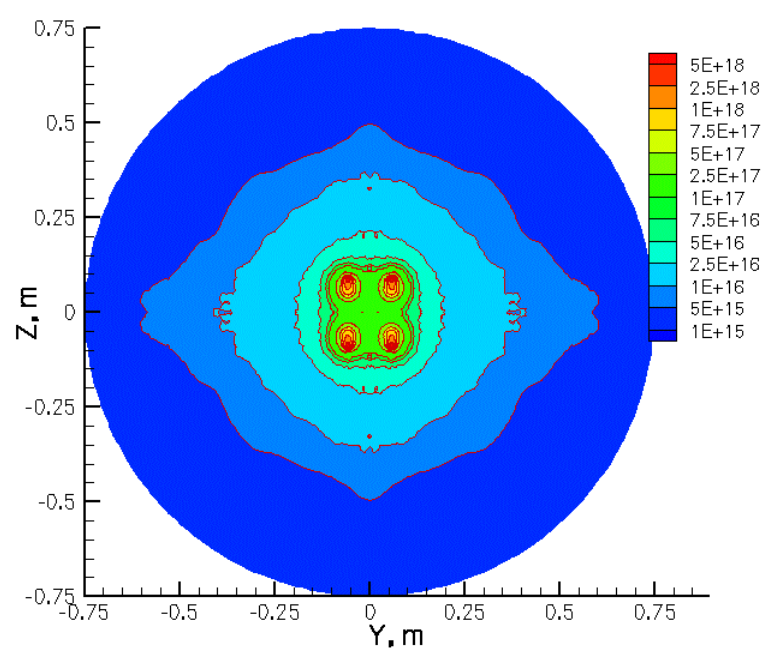

Figure 3. Neutral Number Density $\left(\mathrm{m}^{-3}\right)$ at $\mathbf{x}=\mathbf{0 . 0 2} \mathrm{m}$. function, the plasma potential and the electron temperature.

\section{Simulation and Results}

For this study, due to the setup symmetry, only one thruster is needed in the simulation and the other results are obtained by reflections.

A specific PIC module is implemented in the University of Michigan code MONACO. ${ }^{15}$ An unstructured mesh containing about 560,000 tetrahedrons is generated with the software HyperMesh. ${ }^{16}$ The very detailed geometries of the $7 \mathrm{~mm}$ conic cap and the small cathode are included in the mesh. The cathode exit plane is an orifice with a diameter of $5.08 \times 10^{-4} \mathrm{~m}$. It is a challenge for meshing and the simulation results indicate that the cathode effects may be important. Simulations are performed on a parallel

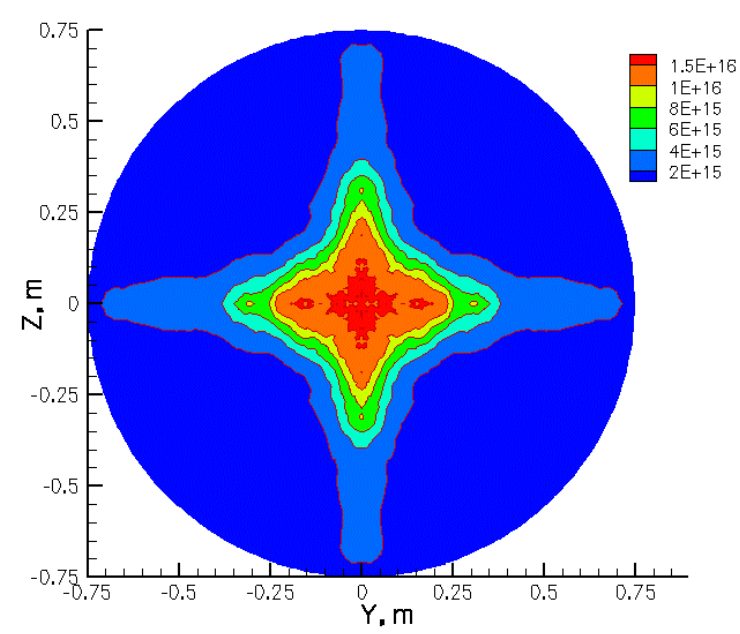

Figure 4. Neutral Number Density $\left(\mathrm{m}^{-3}\right)$ at $\mathrm{x}=0.5 \mathrm{~m}$.

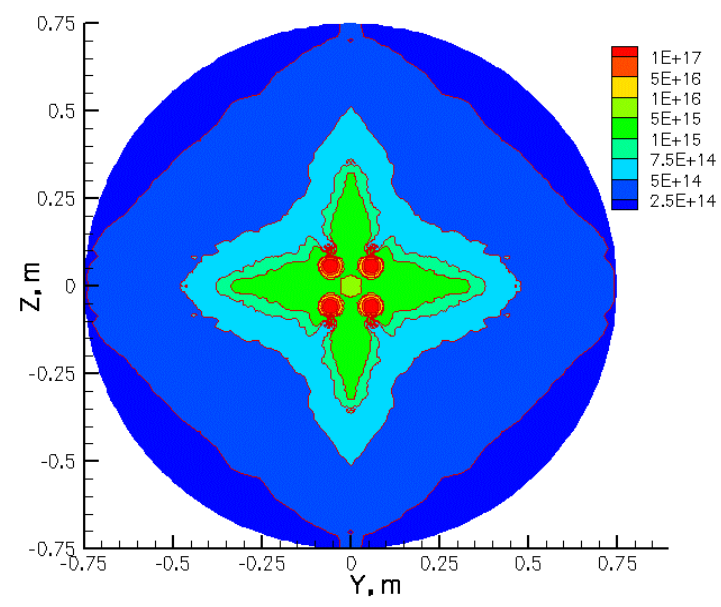

Figure 5. Electron Number Density $\left(\mathrm{m}^{-3}\right)$ at $\mathrm{x}=\mathbf{0 . 0 2 m}$. 
machine with 4 processors and with 5.5 million particles at the final steady state. The finite element solver is called every 10 time-steps for Eqs. $(4,7,8)$ and takes a significant fraction of the simulation time. The whole simulation costs $120 \mathrm{CPU}$ hours with a total of 60,000 time steps.

Figures 3-6 present the neutral and electron number density distributions in the near field $(\mathrm{x}=0.02 \mathrm{~m})$ and far field $(x=0.5 \mathrm{~m})$. For neutral particles, due to the cluster effects, the plumes are similar to flows passing right angle corners where $45^{\circ}, 135^{\circ}, 225^{\circ}$ and $315^{\circ}$ are the major diffusion directions, and there will be concentrations along the centerline. Figures 3 and 4 show smaller number densities along the four specific directions. These effects are also true for ions, and the strong electric interaction among the different plumes results in an evenly distributed far field in Fig.6. The

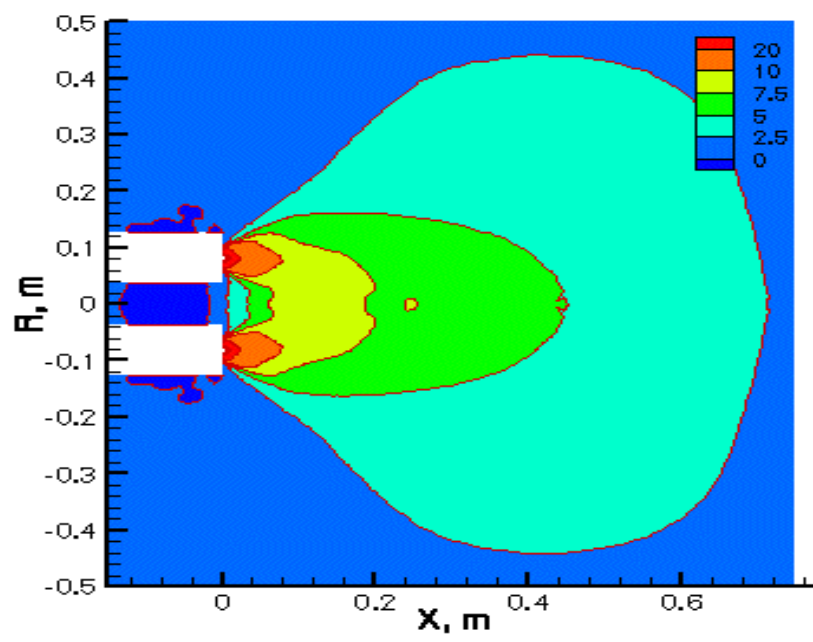

Figure 7. Plasma Potential (V) across Thrusters 2 and 4.

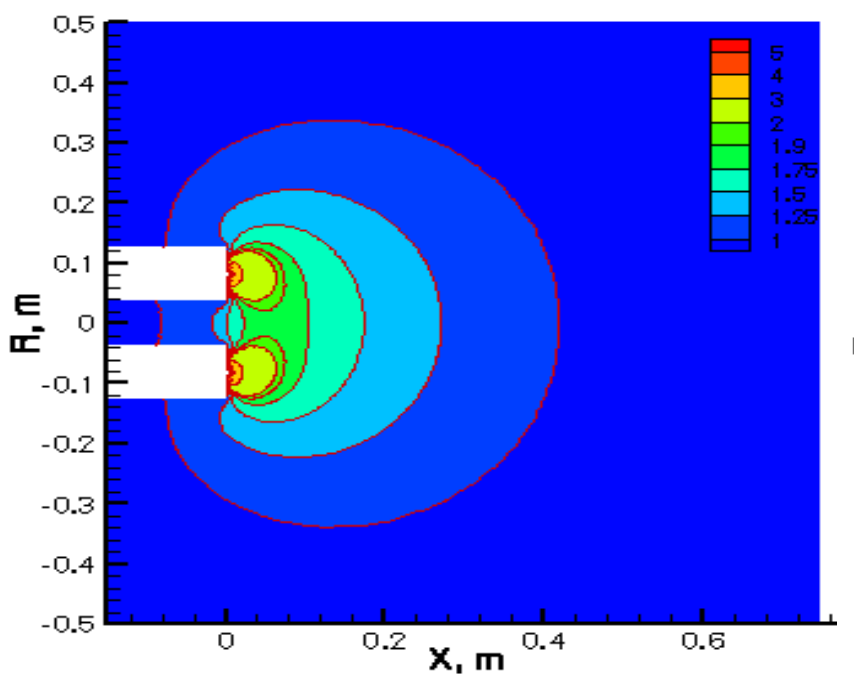

Figure 9. Electron Temperature (ev) across Thrusters 2 and 4.

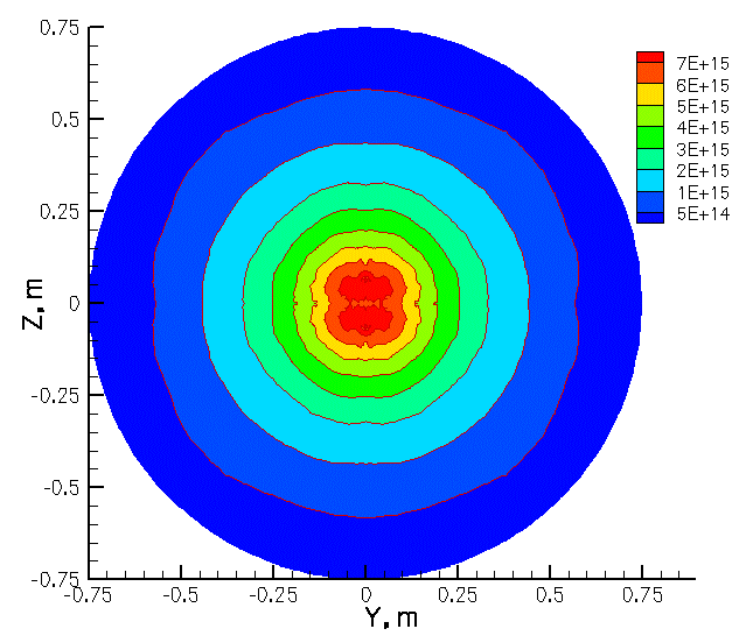

Figure 6. Electron Number Densitv $\left(\mathrm{m}^{-3}\right)$ at $\mathrm{x}=0.5 \mathrm{~m}$.

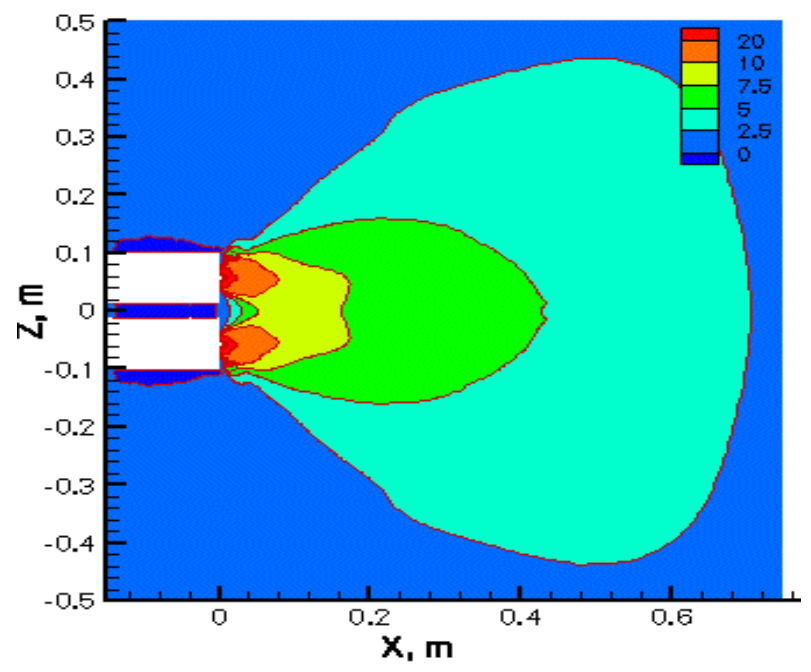

Figure 8. Plasma Potential (V) across Thrusters 3 and 4.

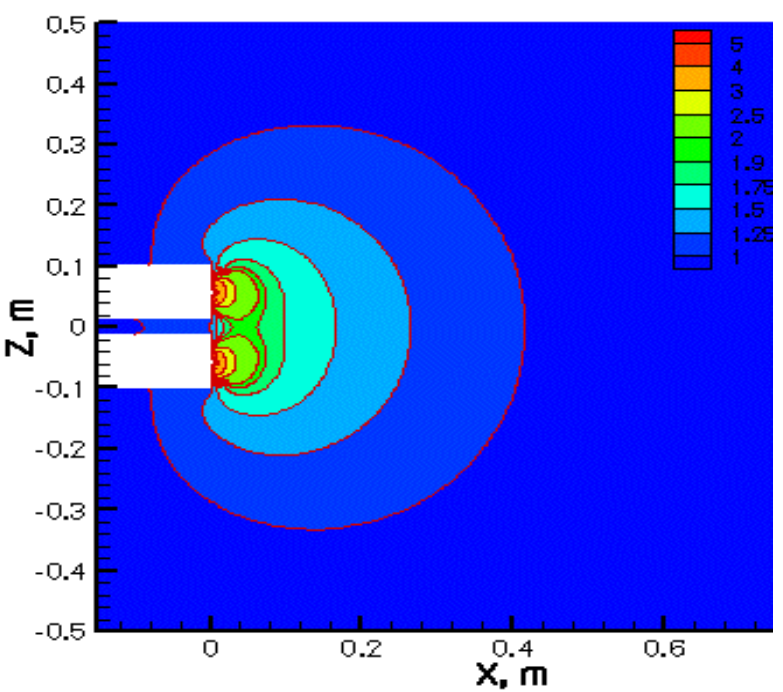

Figure 10. Electron Temperature (ev) across Thruster 3 and 4. 
cathode effects are evident for the near field and there is a small centerline ion concentration in Fig. 5 due to CEX effects. These results indicate very different results across different planes for neutral number density distribution and near field ion number density distribution, such as in the vertical plane across thrusters 3 and 4 and in the plane across thrusters 2 and 4.

Figures 7-10 present contours of plasma potential and electron temperature passing through the centers of thrusters 3 and 4. In the near field, the plumes are well separated while at a short distance from the thruster exit plane they merge into one plume.

Figures 11 and 12 present comparisons of plasma potentials along different centerlines and the station $\mathrm{x}=0.05 \mathrm{~m}$, respectively. It is evident that there is no significant potential difference between the vertical plane passing through thrusters 1 and 4, and the vertical plane passing through the thrusters 3 and 4 . Figure 11 also shows that the plasma potentials along four different centerlines converge into one universal distribution at station $X=0.15 \mathrm{~m}$, which indicates that the four plume flows merge into one. These two figures also indicate that the numerical simulation predicts lower potential peak values than the experimental measurements at specific locations along a thruster centerline and a channel centerline. However, they predict a matching distribution along the centerline passing the middle point of two thrusters, and the general shape of the potential profile at $\mathrm{X}=0.05 \mathrm{~m}$ fit the experimental measurement as well.

Figures 13 and 14 present the electron number densities along different centerlines and at station

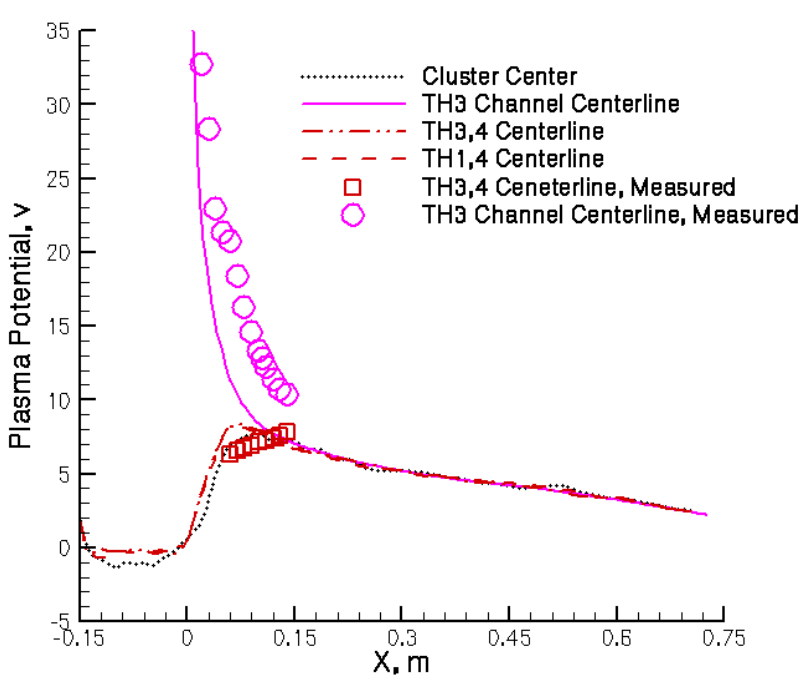

Figure 11. Plasma Potentials along Centerlines.

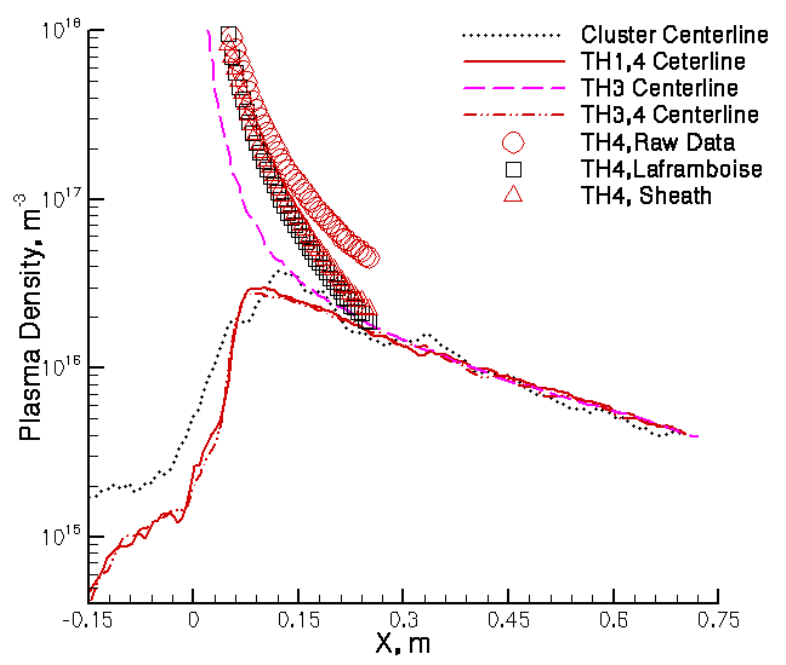

Figure 13. Plasma Number Density along Centerlines.

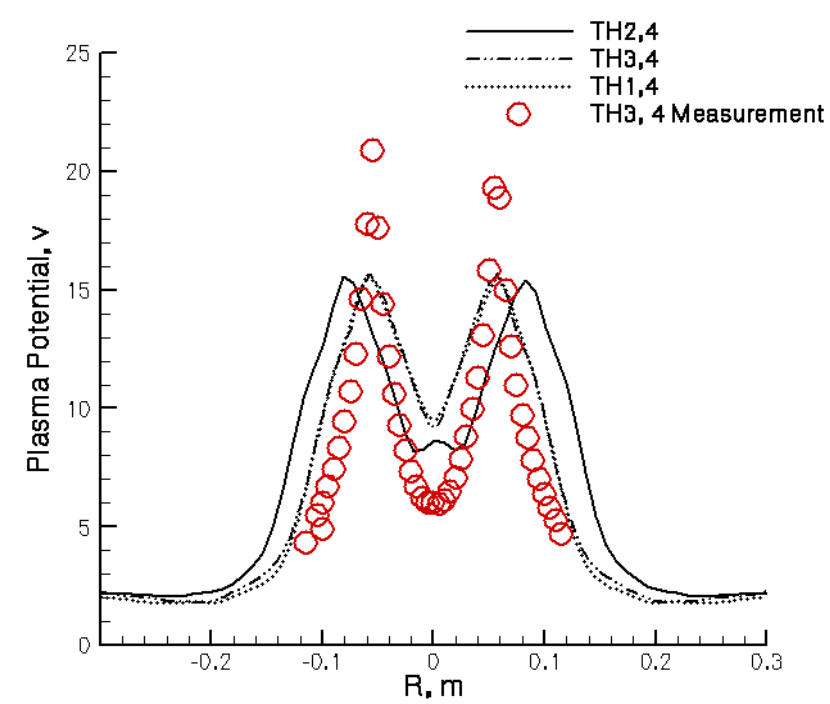

Figure 12. Plasma Potential at Station $X=0.05 m$.

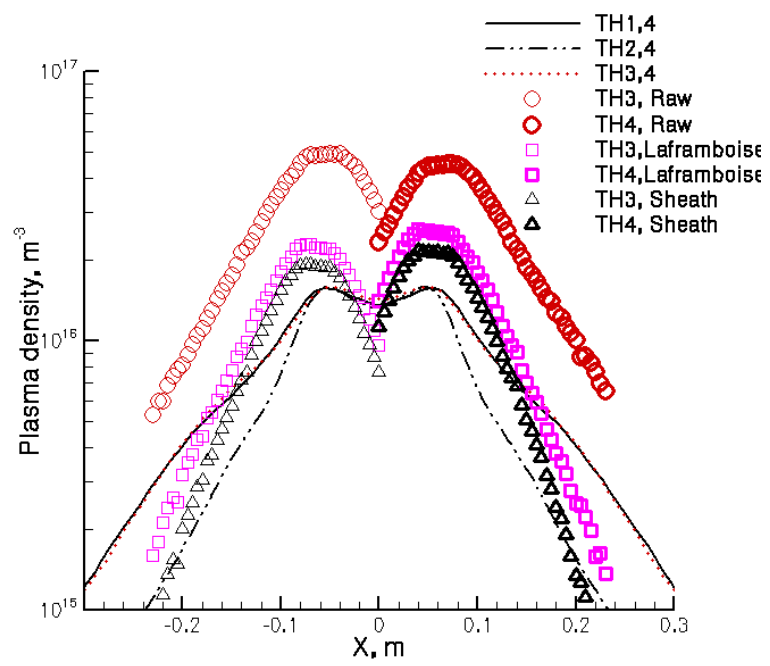

Figure 14. Plasma Number Density at Station $X=0.20 \mathrm{~m}$.

$-10-$

American Institute of Aeronautics and Astronautics Paper 2005-4662 
$\mathrm{X}=0.20 \mathrm{~m}$,respectively. Figure 13 indicates that the number densities from all plumes merge into one at the station around $\mathrm{X}=0.20 \mathrm{~m}$. Electron number density obtained from a Langmuir probe always contains the greatest experimental uncertainty. ${ }^{4}$ Three different profiles of measured data are shown representing two different corrections to the raw data. ${ }^{4}$ The profile labeled "sheath" assumes that the probe collection radius is increased by a sheath of five Debye lengths, thus leading to a reduction in plasma density. The profile labeled "Laframboise" incorporates corrections due to the slightly different voltages applied to each of the three probes in the instrument. The correction is sensitive to the ratio of electron to ion temperature, and a ratio of one is assumed in the corrected data shown here. Comparisons indicate that in general the numerical results predict significantly lower values than the experiments. However, the sheath and Laframboise corrected datayield better agreement with the simulation data.

Figure 15 presents the comparison of electron temperature distributions along different centerlines. It is significant to mention that the Boltzmann relation assumes a constant electron temperature and cannot predict a variation. The detailed fluid electron model yields a non-constant electron temperature field which is more physically reasonable and close to the experimental measurements. The closer to the thruster exit, the higher the electron temperature, and the simulation results predict that the electron temperature from different plumes merges at station $\mathrm{X}=0.05 \mathrm{~m}$, which is much closer to the thrusters compared with the plasma potential and electron number density. The experimental data was obtained with a triple Langmuir probe.

The neutral number density can be predicted with a
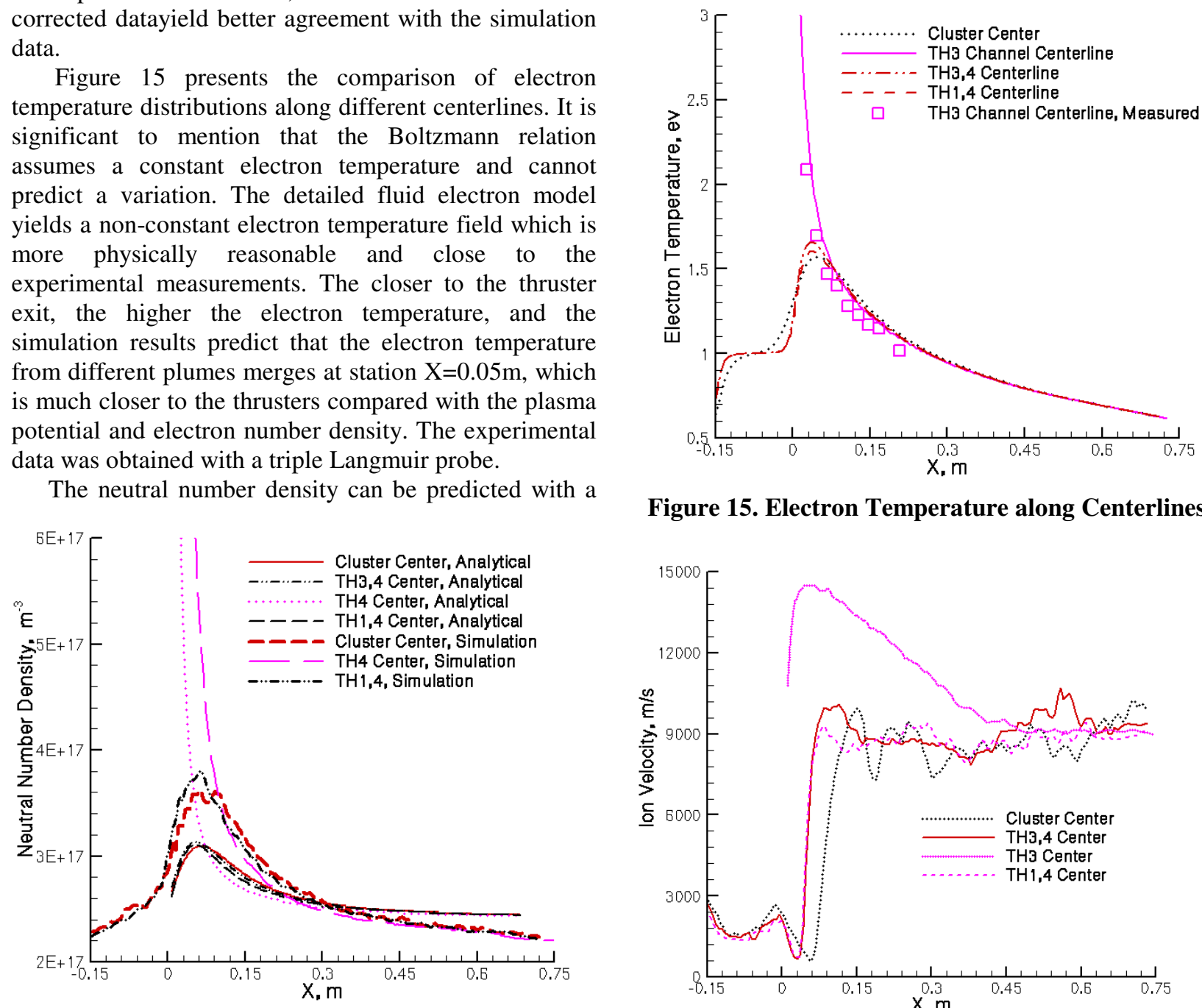

Figure 15. Electron Temperature along Centerlines.

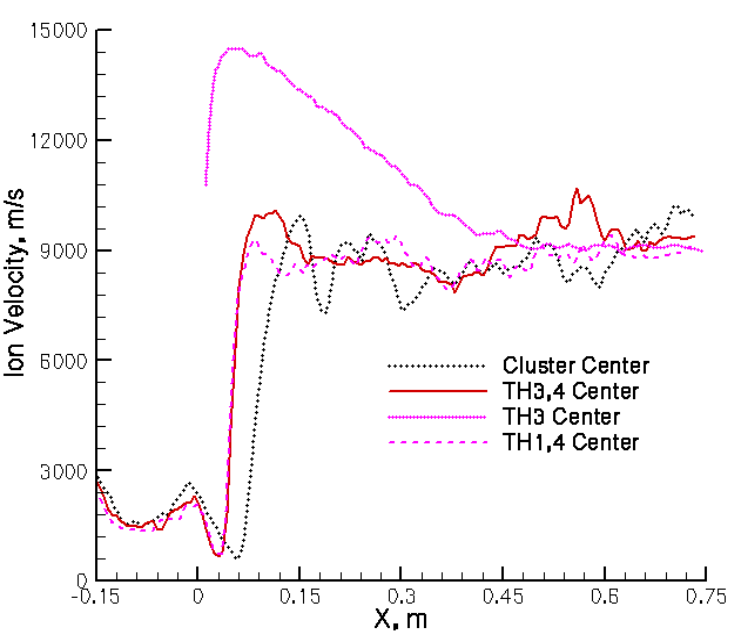

Figure 16. Neutral Number Density along Centerlines.

Figure 17. Ion Velocity along Centerlines. simple free molecular flow model. Because the neutral flow is highly rarefied, the Knudsen number for neutrals is about 55 for a channel height of $7.5 \times 10^{-3} \mathrm{~m}$. Hence the neutral number density distribution can be approximated with a free molecule flow model with zero mean velocity from four small ring sources of anodes and four small orifices of cathodes. At any specific point with coordinates $(x, y, z)$ in front of the thrusters, the velocity phase at that point can only have non zero values within specific solid angles from the four ring sources of anodes and four small orifices of cathodes. The number density distribution at that point can be estimated by the solid angles at that point subtended by these four ring sources and four cathodes. The derivation process is straightforward but tedious and the final expression for the solid angle is listed in the appendix.

The final expression for the number density at any point $(x, y, z)$ in front of the thruster cluster is: 


$$
\begin{aligned}
& n(x, y, z)=n_{\text {back }}+\frac{x\left(R^{2}-r^{2}\right) n_{a}}{4 \pi}\left(F\left(A_{1}, A_{2}\right)+F\left(A_{3}, A_{4}\right)+F\left(A_{5}, A_{6}\right)+F\left(A_{7}, A_{8}\right)\right)+\frac{n_{c}}{4 \pi}\left(H\left(n_{x 1}, n_{y 1}, n_{z 1}, b, a, d\right)\right. \\
& \left.\quad+H\left(n_{x 2}, n_{y 2}, n_{z 2}, b,-a, d\right)+H\left(n_{x 3}, n_{y 3}, n_{z 3}, b,-a,-d\right)+H\left(n_{x 4}, n_{y 4}, n_{z 4}, b, a,-d\right)\right)
\end{aligned}
$$

Figure 16 presents the comparison between the simulation results and the analytical results along different centerlines. Both the simulation results and the analytical results present the same trends: The neutral number density along the cluster centerline and the centerline of vertical/horizontal planes first increases quickly then decreases slowly with a maximum value at a specific distance from the thruster; the maximum value along the cluster centerline is further downstream from the thruster face and is smaller than the value on the centerline between two thrusters. The difference between simulation and analytical results can be explained by the following factors omitted in this crude analytical model: wall effects, the momentum exchange collisions and the CEX change effect and the non-zero mean velocity at the exit planes of anodes and cathodes. Especially, the wall effect is significant in the very near field because the thruster wall area is large. Despite these factors, the comparison still yields a certain agreement.

Figure 17 presents the ion velocities along different centerlines. Along the thruster centerline, the major acceleration region is about $0.05 \mathrm{~m}$ downstream from the thrusters where the major electric potential drop occurs. After that region, the ion velocity drops abruptly due to charge exchange. Figure 18 illustrates how the upward and

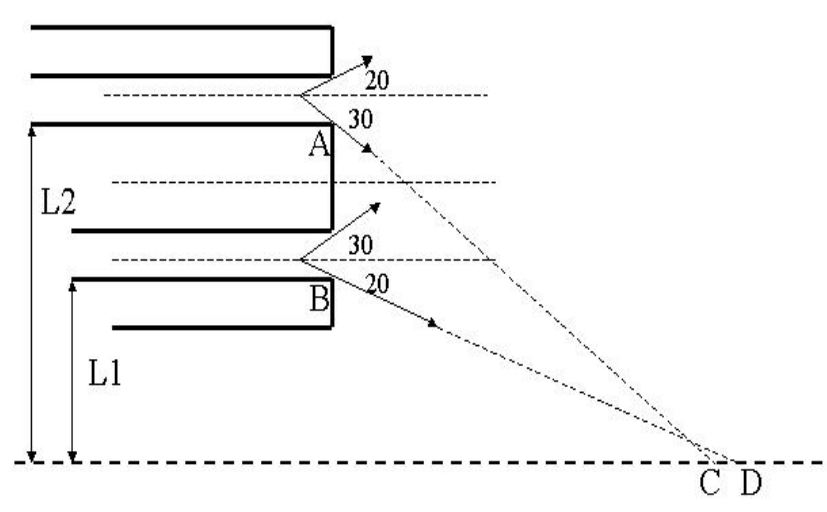

Figure 18. Geometry Effect on Ion Velocity along Centerlines.

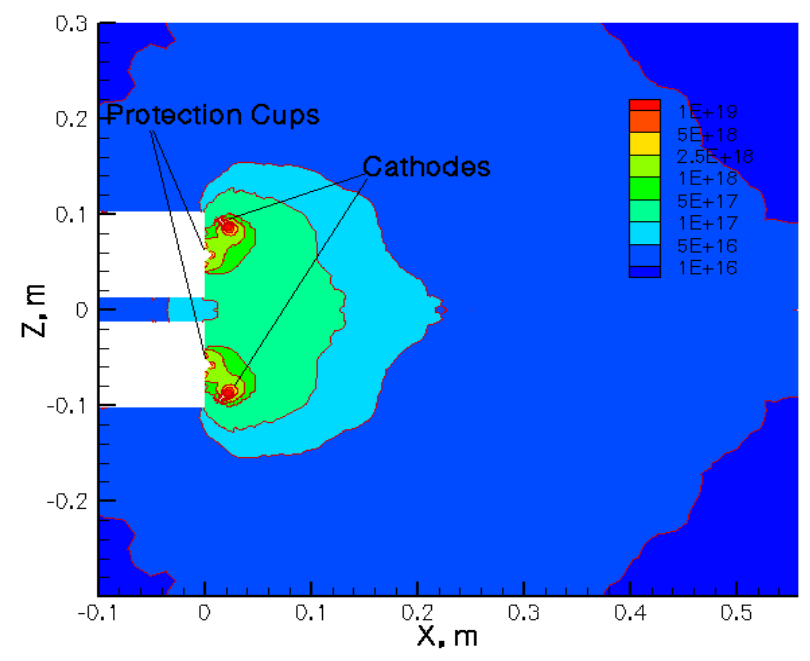

Figure 20. Neutral Number Density across Thrusters 3 and 4.

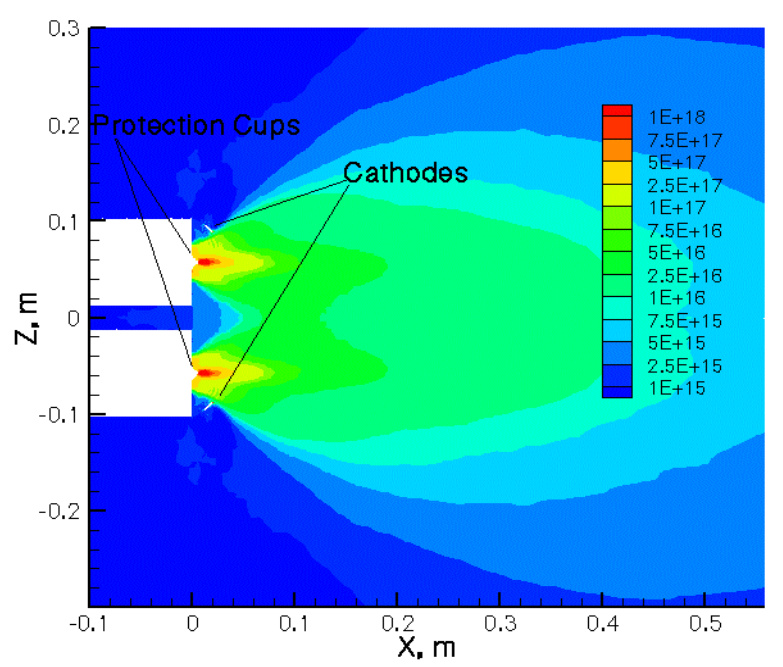

Figure 19. Ion Number Density across Thrusters 3 and 4.

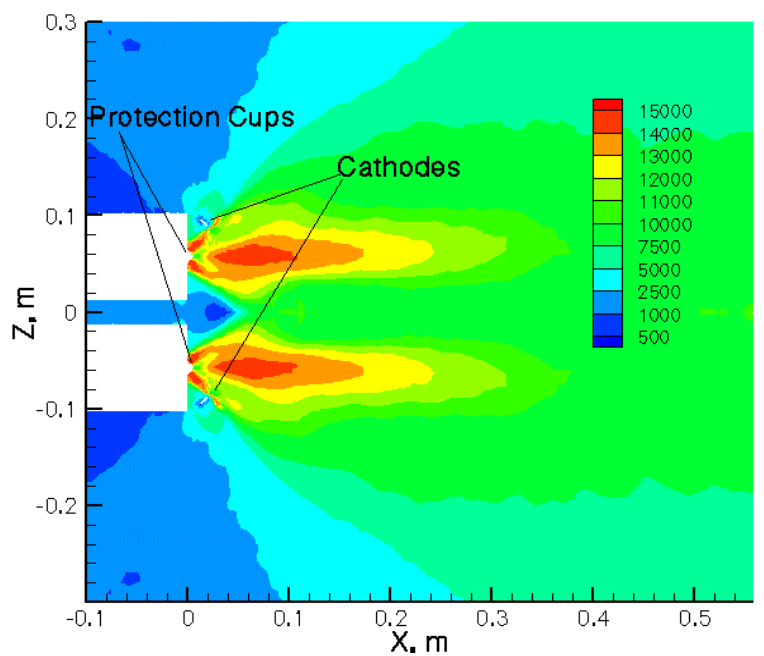

Figure 21. Ion Velocity Contours across Thrusters 3 and 4.

$-12-$

American Institute of Aeronautics and Astronautics Paper 2005-4662 
downward divergence angles have effects on the centerline ion velocities. For example, the first ion beam from points $\mathrm{A}$ and $\mathrm{B}$ will reach points $\mathrm{C}$ and $\mathrm{D}$ correspondingly. At locations on the centerline upstream points $\mathrm{C}$ and $\mathrm{D}$, the ions are most probably created by the CEX effect and possess slow velocities. The calculation for the cluster centerline predicts the first high peak velocity occurs at a location of $X=0.1527 \mathrm{~m}$, and matches the result in Fig. 17 perfectly. After that point, the incoming ion beams from other thrusters strongly interact due to the electric field effects, resulting in several waves in the plume structure reflected in the centerline ion velocity. It also indicates that different from the neutral velocities which merge very quickly at $\mathrm{X}=0.15 \mathrm{~m}$, the ion velocities from different centerlines merge further downstream at station $\mathrm{X}=0.45 \mathrm{~m}$.

The cathode is included in this simulation essentially as a point source located off the thruster wall, while for the axi-symmetric simulation in Ref. 3, the cathode was treated as an annulus on the thruster front face without mass flux. In addition, the protection cap was not included in Ref.3. Due to these differences, though most of the parameters are the same for both simulations, this 3D simulation yields some results that are different from the axisymmetric simulation in Ref. 3.

Figures 19-21 present contours of ion number density, neutral number density and ion velocity across the vertical plane passing through thrusters 3 and 4 . This plane intersects two $7 \mathrm{~mm}$ conic protection caps and two cathodes. Figure 19 shows there is a high ion number density accumulated next to the protection cap, and the two plumes eventually merge into one. Figure 20 indicates that there is a large amount of slow neutrals emitted by the two cathodes, while Fig.21 indicate that these slow neutrals reduce the ion velocity contours through the CEX mechanism. The last two figures indicate that it is essential to include the cathode in a plume flow simulation.

\section{Conclusion}

In this study, a comprehensive 3D DSMC-PIC package with a general purpose finite element solver and a detailed electron fluid model was developed and applied to simulate the plasma plume flow from a cluster of four Hall thrusters. Several major implementation issues were reviewed.

Generally the simulation results matched the experimental measurements or analytical results and the cluster effects are evidently captured. From the results along different centerlines, different properties of the four plumes merge into one plume at different locations from $X=0.05 \mathrm{~m}$ to $X=0.40 \mathrm{~m}$.

The simulation also indicated that the cathodes may have significant effects on the plume flows.

\section{Appendix}

The solid angle at the point $(\mathrm{x}, \mathrm{y}, \mathrm{z})$ subtended by the four ring sources and four cathodes is:

$$
\begin{aligned}
& \Omega=\sum_{i=1}^{4} \int \frac{x}{R_{i}^{3}} d s_{i}+\sum_{j=1}^{4} \int \frac{(x-b) n_{x j}+\left(y-a_{j}\right) n_{x j}+\left(z-d_{j}\right) n_{x j}}{R_{j}^{3}} d s_{j} \\
& =\sum_{i=1}^{4} \int_{0}^{2 \pi} \frac{x}{R_{i}^{3}} \frac{(R-r)(R+r)}{2} d \theta+\sum_{j=1}^{4} \int \frac{(x-b) n_{x j}+\left(y-a_{j}\right) n_{x j}+\left(z-d_{j}\right) n_{x j}}{R_{j}^{3}} d s_{j} \\
& =\frac{x\left(R^{2}-r^{2}\right)}{2} \int_{0}^{2 \pi} \frac{d \theta}{\left(x^{2}+\left(y-\left(a+\frac{R+r}{2} \cos \theta\right)\right)^{2}+\left(z-\left(a+\frac{R+r}{2} \sin \theta\right)\right)^{2}\right)^{3 / 2}} \\
& +\frac{x\left(R^{2}-r^{2}\right.}{2} \int_{0}^{2 \pi} \frac{d \theta}{\left(x^{2}+\left(y-\left(-a+\frac{R+r}{2} \cos \theta\right)\right)^{2}+\left(z-\left(a+\frac{R+r}{2} \sin \theta\right)\right)^{2}\right)^{3 / 2}} \\
& +\frac{x\left(R^{2}-r^{2}\right)^{2 \pi}}{2} \int_{0}^{2 \pi} \frac{d \theta}{\left(x^{2}+\left(y-\left(a+\frac{R+r}{2} \cos \theta\right)\right)^{2}+\left(z-\left(-a+\frac{R+r}{2} \sin \theta\right)\right)^{2}\right)^{3 / 2}} \\
& +\frac{x\left(R^{2}-r^{2}\right)^{2 \pi}}{2} \int_{0}^{2 \pi} \frac{\left(x^{2}+\left(y-\left(-a+\frac{R+r}{2} \cos \theta\right)\right)^{2}+\left(z-\left(-a+\frac{R+r}{2} \sin \theta\right)\right)^{2}\right)^{3 / 2}}{+H\left(n_{x 1}, n_{y 1}, n_{z 1}, b, a, d\right)+H\left(n_{x 2}, n_{y 2}, n_{z 2}, b,-a, d\right)+H\left(n_{x 3}, n_{y 3}, n_{z 3}, b,-a,-d\right)} \\
& +H\left(n_{x 4}, n_{y 4}, n_{z 4}, b, a,-d\right) \\
& =x\left(R^{2}-r^{2}\right)\left(F\left(A_{1}, A_{2}\right)+F\left(A_{3}, A_{4}\right)+F\left(A_{5}, A_{6}\right)+F\left(A_{7}, A_{8}\right)\right)+H\left(n_{x 1}, n_{y 1}, n_{z 1}, b, a, d\right)
\end{aligned}
$$


where

$$
+H\left(n_{x 2}, n_{y 2}, n_{z 2}, b,-a, d\right)+H\left(n_{x 3}, n_{y 3}, n_{z 3}, b,-a,-d\right)+H\left(n_{x 4}, n_{y 4}, n_{z 4}, b, a,-d\right)
$$

$$
\begin{gathered}
H\left(n_{x}, n_{y}, n_{z}, b, a, d\right)=\left\{\begin{array}{r}
\frac{\pi R_{c}^{2}\left((x-b) n_{x}+(y-a) n_{y}+(z+b) n_{z}\right)}{\left((x-b)^{2}+(y-a)^{2}+(z+d)^{2}\right)^{3 / 2}},(x-b) n_{x}+(y-a) n_{y}+(z-d) n_{z}>0 \\
0, \quad(x-b) n_{x}+(y-a) n_{y}+(z-d) n_{z} \leq 0
\end{array}\right. \\
F\left(A_{i}, A_{j}\right)=E\left(\sqrt{\frac{2 A_{j}}{A_{i}+A_{j}}}\right) /\left(A_{i}+A_{j}\right)^{3 / 2}, E(x) \text { is the complete elliptical integrals, and } \\
A_{1}=(y-a)^{2}+\left(\frac{R+r}{2}\right)^{2}+x^{2}+(z-a)^{2}, A_{2}=(R+r) \sqrt{(y-a)^{2}+(z-a)^{2}} \\
A_{3}=(y+a)^{2}+\left(\frac{R+r}{2}\right)^{2}+x^{2}+(z-a)^{2}, A_{4}=(R+r) \sqrt{(y+a)^{2}+(z-a)^{2}} \\
A_{5}=(y-a)^{2}+\left(\frac{R+r}{2}\right)^{2}+x^{2}+(z+a)^{2}, A_{6}=(R+r) \sqrt{(y-a)^{2}+(z+a)^{2}} \\
A_{7}=(y+a)^{2}+\left(\frac{R+r}{2}\right)^{2}+x^{2}+(z+a)^{2}, A_{8}=(R+r) \sqrt{(y+a)^{2}+(z+a)^{2}}
\end{gathered}
$$

The above derivation process uses the elliptical calculus formula ${ }^{17}$ :

$$
\int_{-\pi / 2}^{\pi / 2} \frac{d \theta}{(a+b \sin \theta)^{3 / 2}}=\frac{2}{(a+b)^{3 / 2}} E\left(\sqrt{\frac{2 b}{a+b}}\right)
$$

\section{Acknowledgments}

This work is supported by the Air Force Office of Scientific Research through Grants F49620-03-1-0123 and FA9550-05-1-0042. The authors gratefully acknowledge the contributions to this work by Dr. Quanhua Sun for discussions and Dr. Brian Beal for sharing his experimental data.

Table 1. Boundary Conditions for the Detailed Electron Fluid Model

\begin{tabular}{|l|l|l|l|l|l|}
\hline Boundary & Outflow & Wall & Anode & Cathode & Symmetric plane \\
\hline$\psi\left(m^{-1} s^{-1}\right)$ & $\frac{\partial^{2} \psi}{\partial n^{2}}=0$ & 0 & $\frac{\partial \psi}{\partial n}=\frac{J_{A}}{e}$ & $\frac{\partial \psi}{\partial n}=\frac{J_{c}}{e}$ & $\frac{\partial \psi}{\partial n}=0$ \\
\hline$\phi(\mathrm{V})$ & 2 & 0 or by Eq. (17) & 93.0 & 7 & $\frac{\partial \phi}{\partial n}=0$ \\
\hline$T e(\mathrm{ev})$ & 0.6 & 1 & 6.0 & 2 & $\frac{\partial T e}{\partial n}=0$ \\
\hline
\end{tabular}

\section{References}

${ }^{1}$ Bird, G.A., Molecular Gas Dynamics and the Direct Simulation of Gas Flows, Clarendon Press, New York, 1991.

${ }^{2}$ Birdsall, C.K., and Langdon, A.B., Plasma Physics Via Computer Simulation, Adam Hilger, New York, June 1991.

${ }^{3}$ Boyd, I. D. and Yim, J.T., "Modelling of the Near Field Plume of a Hall Thruster", Journal of Applied Physics, Vol. 95,2004, pp.4575-4584.

${ }^{4}$ Hargus, W.A. and Reed, G., "The Air Force Clustered Hall Thruster Program”, AIAA Paper 2002-3678, July 2002, Indianapolis, IN.

${ }^{5}$ Beal, B.E., "Clustering of Hall Effect Thrusters for High-Power Electric Propulsion," Ph.D. Dissertation, Aerospace Engineering Dept., the Univ. of Michigan, Ann Arbor, MI, 2004.

${ }^{6}$ Ahedo, E., Martinez-Cerezo, P., and Martinez-Sanchez, M., "One-dimensional Model of the Plasma Flow in a Hall Thruster", Physics of Plasma, Vol. 8, 2001, pp.3058-3068.

${ }^{7}$ Mitcher, M., and Kruger, C.H., "Partially Ionized Gases", Wiley, 1973.

${ }^{8}$ Vichnevetsky, R., “Computer Methods for Partial Differential Equations”, Prentice-Hall, New Jersey 1981.

${ }^{9}$ Press, W.H., Teukolsky, S.A., Vetterling, W.T. and Flannery, B.P., Numerical Recipes in $C, 2^{\text {nd }}$ ed., Cambridge University Press, Cambridge, UK, 1999. 
${ }^{10}$ Hammel, J.R., "Development of An Unstructured 3-D Direct Simulation Monte Carlo/Particle-In-Cell code and the Simulation of Microthruster Flows," M.S. Dissertation, Mechanical Engineering Dept., Worcester Polytechnic Institute, Worcester, MA, 2002.

${ }^{11}$ Ruyten, W.M., "Density-conserving shape factors for particle simulations in cylindrical and spherical coordinates," Journal of Computational Physics," Vol. 105, 1993, pp.224-232.

${ }^{12}$ Dalgarno, A., McDowell, M.R.C, and Williams, A., "The Mobilities of Ions in Unlike Gases," Proceedings of the Royal Society, Vol. 250, 1958, pp411-425.

${ }^{13}$ Pullins, S.H., Chiu, Y., Levandier, D.J. and Dresseler, R.A., "Ion Dynamics in Hall Effect and Ion Thruster -Xenon Symmetric Charge Transfer," AIAA Paper 2000-0636, Jan. 2000, Reno, NV.

${ }^{14}$ Miller, S., Levandier, D.J., Chiu, Y., and Dressler, R.A., "Xenon Charge Exchange Cross Sections for Electrostatic Thruster Models," Journal of Applied Physics, Vol. 19, 2002, pp.984-991.

${ }^{15}$ Dietrich, S. and Boyd, I. D., "Scalar and Parallel Optimized Implementation of the Direct Simulation Monte Carlo Method," Journal of Computational Physics, Vol. 126, p. 328, 1996

${ }^{16}$ HyperMesh, Ver.6.0, Altair Engineering Inc., Troy, MI, 2003.

${ }^{17}$ Byrd, P. and Friedman, M., Handbook of Elliptic Integrals For Engineers and Physicists, Springer-Verlag, 1954, pp173. 